\title{
Regulatory T cells are present in Kaposi's sarcoma and increasingly frequent in advanced disease D Camarillo1 ${ }^{1}$, K Leslie* ${ }^{*}$, P Unemori ${ }^{1}$, A Koba Yashi² ${ }^{2}, \mathrm{~K} \mathrm{McCune-Smith}^{2}$ and T Maurer $^{1}$
}

Address: ${ }^{1}$ Department of Dermatology, University of California, San Francisco, California, USA and ${ }^{2}$ Department of Obstetrics and Gynecology, University of California, San Francisco, California, USA

* Corresponding author

from II th International Conference on Malignancies in AIDS and Other Acquired Immunodeficiencies (ICMAOI): Basic, Epidemiologic, and Clinical Research

Bethesda, MD, USA. 6-7 October 2008

Published: 17 June 2009

Infectious Agents and Cancer 2009, 4(Suppl 2):PI2 doi:I0.II86/I750-9378-4-S2-PI2

This abstract is available from: http://www.infectagentscancer.com/content/4/S2/PI2

(c) 2009 Camarillo et al; licensee BioMed Central Ltd.

\section{Background}

Regulatory T cells (Tregs) are thought to play a crucial role in preventing and controlling hyperactive immune responses in malignancies and inflammatory disease. The role of Tregs in Kaposi's sarcoma (KS) has not been explored and is of particular interest in that this disease demonstrates characteristics of both inflammation and malignancy. We examined the presence and frequency of Tregs in KS tissue samples compared to normal skin. In addition, we compared presence and frequency of Tregs across three distinct histopathologic stages of KS. The histopathologic comparisons included the earlier patch and plaque stages, and the most advanced, nodular stage of KS.

\section{Methods}

Regulatory $\mathrm{T}$ cells are characterized by the expression of FOXP3, CD4 and CD25. A total of 15 cutaneous KS samples were obtained from different patients. Of the total 15 KS samples, breakdown of histopathologic subtypes included 6 samples from patches, 4 samples from plaques, and 5 samples from nodules. Eight samples from normal skin served as controls. Immunohistochemical and immunofluorescent assays and image analysis were performed on all samples.

\section{Results}

The frequency of FOXP3+ cells in all stages of KS was significantly higher compared to normal skin with means \pm standard deviation of $43.4 \pm 47.8$ FOXP3 + cells $/ \mathrm{mm}^{2}$ in KS versus $4.56 \pm 12.5$ FOXP $3+$ cells $/ \mathrm{mm}^{2}(\mathrm{p}<0.001)$. This difference remained significant when comparing each individual stage to normal skin ( $\mathrm{p}<0.001$ for nodular and plaque stages versus normal skin, $\mathrm{p}=0.002$ for patch stage versus normal skin). The number of FOXP3 + cells was highest in the nodular stage $70.3 \pm 47.8$ FOXP3+ cells/ $\mathrm{mm}^{2}$ compared to both patch $29.3 \pm 50.8 \mathrm{p}=0.001$ and plaque $27.8 \pm 33.2 \mathrm{p}<0.001$ stages. The frequency of FOXP3+ cells in patch and plaque stages was not significantly different.

\section{Conclusion}

Tregs are present in KS and are increasingly frequent in advanced disease. This finding of increased Tregs in the most advanced stage of KS is a phenomenon that has also been demonstrated in other malignancies including melanoma, ovarian cancer, and hepatocellular carcinoma. Tregs might play a critical role in suppressing the KS-specific immune response and contributing to the unchecked proliferation that is characteristic of KS. In addition, our finding that Tregs were markedly increased in the most advanced stage of KS suggests that regulatory $\mathrm{T}$ cells may also play a key role in KS progression. Ultimately, targeted regulatory $\mathrm{T}$ cell immunotherapy may lead to improved treatment response and prognosis. 